\section{Mental Representations of Values and Behaviors} Do
European Journal of Personality $0(0) 1-16$ (C) The Author(s) 2021

Article reuse guidelines: sagepub.com/journals-permissions DOI: $10.1177 / 08902070211034385$ journals.sagepub.com/home/ejop

(SAGE

\author{
Gabriel Lins De Holanda Coelho ${ }^{1,2}\left(\mathbb{D}\right.$, Paul H. P. Hanel ${ }^{1,3,4}{ }^{\mathbb{D}}$, \\ Mark K. Johansen' and Gregory R. Maio',3
}

\begin{abstract}
The present research provides the first direct assessment of the fit of diverse behaviors to putatively related personal and social values from Schwartz's theory. Across three studies, we examined spatial representations of value-related behaviors that were explicitly derived from people's mental representations of the values. Participants were asked how similar the behaviors were to each other and various values, and these judgments were used to specify multidimensional scaling solutions. The results indicated that the spatial representation of the behaviors was consistent with the twodimensional space described in Schwartz's model of values, although several deviations occurred. For example, selfenhancement behaviors were widely spread, indicating more variation in the way individuals interpret these behaviors, which are often associated with other value types. These data provide evidence that a range of behaviors can at least partly be reduced to underlying motivations expressed by values. Furthermore, our findings indicate that behaviors are often expressed by several values, which might help to explain why value-behavior associations in previous studies were weak. Finally, they illustrate a new approach to learning which behaviors might relate to multiple values.
\end{abstract}

\title{
Keywords
}

behaviors, human values, Schwartz, similarity judgments

Received I8 August 2020; Revised 22 June 202I; accepted 2 July 202 I

The link between values and behaviors is axiomatic in a range of real-world contexts. For instance, the Department for Education in Britain has emphasized the importance of teaching of British values, including democracy, law, liberty, and mutual respect and tolerance, allowing students to challenge opinions or behaviors that oppose these principles (Adams, 2014). Similarly, the G20 group of nations recently initiated annual Values ("V20") summits to explicitly consider common values among the G20 nations and the actions they express (https:// values20.org/).

These assumptions about the importance of values are reinforced by research examining connections between values and behaviors (Bardi \& Schwartz, 2003; Boer \& Fischer, 2013; Lee et al., in press; Rokeach, 1973; Skimina et al., 2019). For instance, values significantly predict attitudes toward immigrants and political activism (Schwartz, 2007). Even research on artificial intelligence has examined values and behavior: When reading stories, an artificial intelligence can learn about the culture where the story is originated (Riedl \& Harrison, 2016). However, similar to conceptually related constructs in social psychology (e.g., personality traits, attitudes; Maio et al., 2018), the associations between values and behaviors depend on myriad contextual factors. Also, values may be used as rationalizations of behavior after the fact (Eiser, 1987; Haidt, 2001; Kristiansen \& Hotte, 1996; Maio, 2016). Furthermore, despite the long-standing interest in value-behavior connections, empirical studies of these connections are far fewer than one might expect (Fischer, 2017).

One explanation for this paucity is the complexity of value-behavior connections. Multiple values may influence any given behavior, and single values may

\footnotetext{
'School of Psychology, Cardiff University, Cardiff, UK

${ }^{2}$ School of Applied Psychology, Cork Enterprise Centre, University College Cork, Cork, Ireland

${ }^{3}$ Department of Psychology, University of Bath, Bath, UK

${ }^{4}$ Department of Psychology, University of Essex, Colchester, UK

Corresponding author:

Gabriel Lins De Holanda Coelho, School of Applied Psychology, Cork Enterprise Centre, University College Cork, Cork, Ireland.

Email: linshc@gmail.com
} 
influence a multitude of behaviors. In addition, these links may occur across varied situations (Schwartz, 1992). Consider behaviors related to the value of freedom. This value can be expressed as behaviors when animal-rights activists are trying to save animals trapped in zoos or circuses or when citizens strive for a region's independence from a perceived occupier. However, behaviors in both examples can also be expressions of additional values: Saving animals may also help protect the environment or even wealth (through loss of animalrelated tourism). Also, regional independence may be perceived as a threat to values of peace or national security. Thus, a key problem in examining the interrelations between values and behaviors is the difficulty of conceptually linking specific values to specific behaviors.

To help decompose this complex problem, the present research introduces the direct mapping of relations between values and behaviors. More specifically, we asked participants to judge the similarity between specific values and behaviors, thereby providing their mental representations (i.e., the characterization of an object within a cognitive system; Hubbard, 2007). For instance, one might think of donating money to charity as a behavior guided by social justice. However, others might think of it as an act promoting social recognition. Thus, our approach allows the mapping of diverse putative interconnections between values and a range of specific behaviors, identifying which behaviors are conceptually close to which values and how their relations can be better assessed.

\section{Theory of Basic Human Values}

Schwartz's (1992, 2012) value model, which has received empirical support in over 80 countries, arranges values in a two-dimensional spatial representation (see Figure 1). This circular organization covers basic motivations expressed through 57 value items. In the original model (Schwartz, 1992), 10 motives are delineated (see Figure 1) as the 10 value types; in turn, these 10 types can be ordered along two dimensions, resulting in four higher order value types. The first dimension contrasts openness to change with conservation motives. The second dimension contrasts self-enhancement with self-transcendence motives.

A core feature of Schwartz's (1992) model is its inclusion of motivational synergies and conflicts. Values placed next to each have more compatible motivations, whereas those further apart are more incompatible. For example, universalism values are adjacent to benevolence values, because they represent and reinforce concern for the welfare of others. In contrast, achievement and power represent motivations for self-enhancement. This motivational continuum has important implications for variables external to the model, including value-relevant behaviors. For example, suppose one behavior exhibits a strong positive correlation with ratings of the importance of one of the value types. In that case, the model

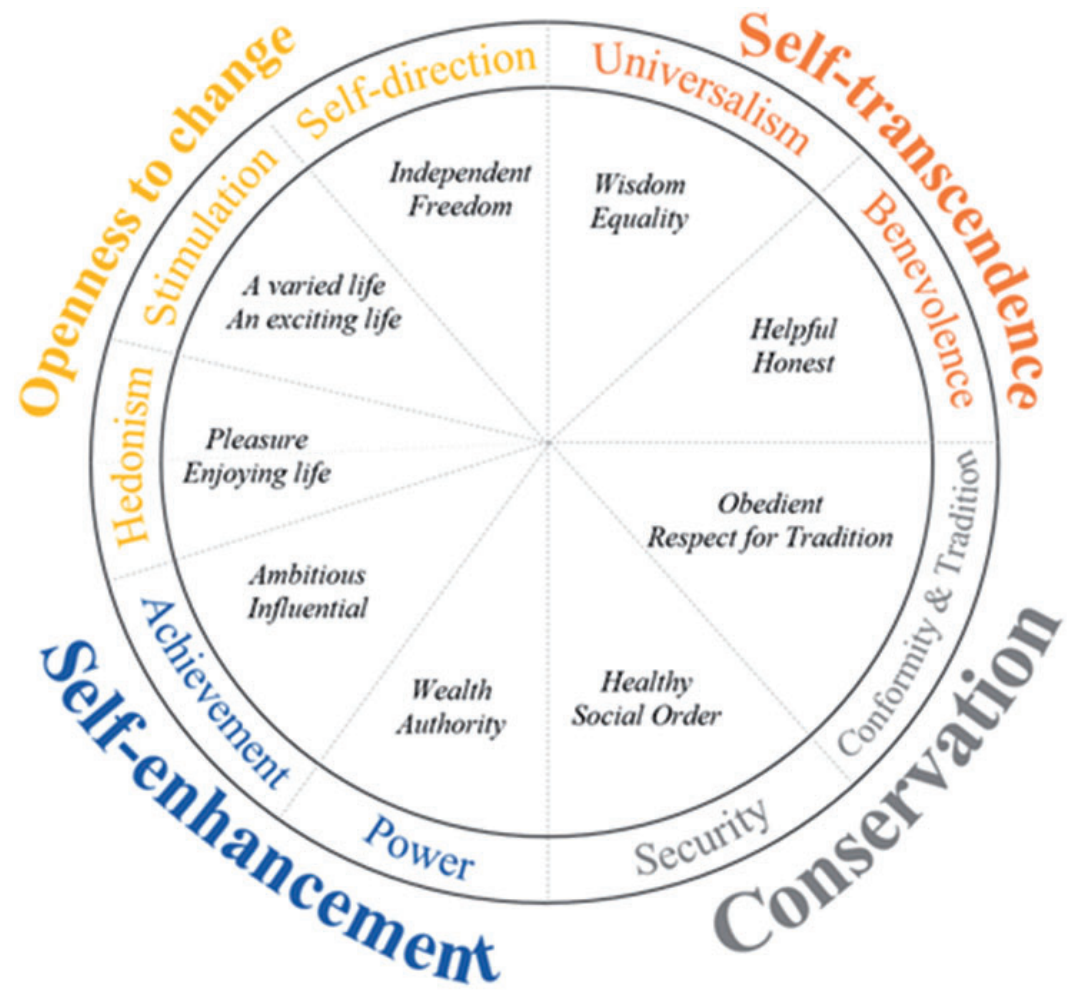

Figure I. Schwartz's (1992) Values Structure (Examples of Values in Italic; Reprinted From Coelho, Hanel, Johansen, \& Maio, 20I8, With Permission). 
predicts that judgment correlations should become progressively less positive (and then perhaps negative), moving around the circular model from adjacent value types through orthogonal value types to opposing value types. This pattern of correlations corresponds to a sinusoidal wave that increases (correlations between a value type and its adjacent values are positive), then decreases (correlating with opposing values are negative), and finally increases again (completing the circle, returning to the adjacent values with positive correlations; e.g., Boer \& Fischer, 2013; Hanel, Zacharopoulos, et al., 2017; Schwartz, 1992). For instance, if individuals who value stimulation (e.g., an exciting life) are more likely to engage in activities that require more physical challenge and danger, the performance of these activities should become progressively less strongly linked to values when moving from adjacent selfdirection and hedonism values through to the opposing values of conformity, security, and tradition (potentially exhibiting negative relations with these latter values). However, such sinusoidal patterns do not always emerge - the type of value itself matters, particularly in terms of values related behaviors.

\section{Assessing Values and Behaviors}

Everyday behaviors associated with values might be represented in a similar space to their values, but to varying degrees. For instance, Bardi and Schwartz (2003) found that correlations between values and behaviors depend on the value type being examined. For example, the frequency with which participants performed behaviors extracted from the value types of tradition (e.g., observe traditional customs on holidays) and stimulation (e.g., do unconventional things) was highly correlated with the importance participants attributed to the behaviors' respective values (e.g., devout, a varied life). In contrast, some values and behaviors deviated from the predictions: For example, conformity values did not correlate with their respective behaviors, but correlated with behaviors from tradition values, an adjacent value type. When assessing the correlations between the behaviors and the 10 value types, with the 10 value types plotted on the $x$-axis and the strength of the correlations on the $y$-axis, the sine wave fit was worst for behaviors derived from universalism and benevolence, but was good for all other behaviors, especially from tradition, stimulation, and hedonism (Hanel, Zacharopoulos, et al., 2017). In a subsequent project, Schwartz and Butenko (2014) attempted to map values and behaviors together. Of the 19 value types, 18 showed stronger associations with their respective behaviors than with others. Interestingly, in both of these studies, the spatial representation between values and behaviors broadly replicated Schwartz's motivational continuum with its agreement and conflict assumptions (Bardi \& Schwartz, 2003; Schwartz \& Butenko, 2014).

Notwithstanding the broad support for the model from these studies, the deviations highlight a critical obstacle in value-behavior research: the complexity of selecting value-expressive behaviors. For example, some behaviors may be more strongly related to other value types than the value type from which they were initially derived. In addition, individuals can classify everyday actions of every sort at different levels of abstraction (Vallacher \& Wegner, 1987, 2014). For instance, in the context of Schwartz's model forgiving someone who insulted you may be categorized as forgiveness or more abstractly as "benevolence" (encompassing forgiveness, helpfulness and other ideals), or even more abstractly as "self-transcendence" (encompassing universalism values). These multiple interpretations for specific actions have been a limitation in psychological studies that involve human behaviors, because of the variability across individuals. Therefore, it is important to consider levels of categorization to obtain reliable representations of what the actors are doing (Vallacher \& Wegner, 1987, 2014).

\section{Value Instantiations: Linking Values and Behaviors}

One approach that might help to link values and behaviors was proposed by Maio $(2010,2016)$, who argued that values are mental representations of ideals that can be examined at three levels: (a) a system level, which is about the relations of the values with each other, as in Schwartz's (1992) model; (b) an abstract level, in which values are construed in terms of feelings and cognitions across situations and relating to the importance that individuals give to abstract value concepts (e.g., pleasure, social order); and (c) an instantiation level, where a value is conceptualized in terms of concrete judgments and actions specific to situations. In Maio's (2010, 2016) system level, individuals may spontaneously group values in a way that reflects their experience. For example, consider an individual who has experienced situations wherein equality was related to others' welfare (e.g., fair income distribution, respect in the workplace for people's differences). This individual is likely to group equality with other values that promote the welfare of others, such as social justice, helpful, and honest, and more distant from other values serving opposing motivations, such as wealth or national security.

At the abstract level, mental representations may also vary. For example, Aavik and Dobewall (2017) assessed whether health, defined in Schwartz's model as the avoidance of disease, could be more broadly represented as different types of health (e.g., mental health, physical health, social health). Using a large sample $(N=1,818)$, Aavik and Dobewall found that the health subcomponents were spread across a third 
dimension. These values were not placed in the openness to change versus conservation dimension, nor the self-enhancement versus self-transcendence, but rather working together with other value types and not limited to the location of the original value (conservation).

At the instantiation level, any value can circumscribe numerous behaviors (cf. Kruglanski et al., 2013), and a priori knowledge about whether a value can predict a behavior requires knowing which behaviors are the strongest instantiations of particular values (Hanel, Vione, et al., 2017). The importance of this approach is shown in past research (Maio et al., 2009) that exposed participants to stories about typical instances of behavior violating the value of equality (e.g., discrimination against women) or atypical instances (e.g., discrimination against lefthanded people). Participants who thought about typical instances exhibited less discrimination in a subsequent experimental task than participants who thought about equality in the context of atypical instances, which did not differ from the control condition. The results supported the hypothesis that the impact of value instantiations on subsequent behavior depends on the extent to which the instantiations are typical exemplars of the value (Maio et al., 2009).

Therefore, it is essential to assess how the connections between values and behavioral instantiations of values occur and assess how these instantiations are mentally represented in connection to the values. In other words, how does a behavior serve as an instance of a value? Not using a behavior that is a typical example of a specific value can result in weak associations (Maio et al., 2009). One approach that has been used in value-behavior research is first to ask individuals of a given culture to provide a list of what they consider to be typical examples of behaviors of a set of values (e.g., Bardi \& Schwartz, 2003; Schwartz \& Butenko, 2014). For instance, research in Brazil indicated that typical behaviors that express the value of "pleasure" include being on holiday at the beach or playing football on a Sunday (cf. Hanel et al., 2018). This approach is the basis for our methods in the present research.

\section{Conceptual Similarities}

Research in cognitive psychology has more frequently used the direct measurement of perceived conceptual similarity between items to identify the structure of mental representations, especially in categorization tasks (see summaries in Murphy, 2004; Oden, 1987). In these tasks, judgments of a new example's similarity to previously known representations (e.g., a single prototype or a set of examples) can be used as a basis for predicting the assignment of the instance to categories. This direct assessment of conceptual representations has been used in two recent sets of studies, one re-examining Schwartz's (1992) value structure
(Coelho et al., 2019), and another (Koch et al., 2016) improving a model of stereotype content (Fiske et al., 2002).

When compared to approaches that involve plotting correlations between ratings of constructs (e.g., values and behaviors; Bardi \& Schwartz, 2003; Schwartz \& Butenko, 2014), the use of similarities provides an alternative and arguably more fundamental assessment of the mental representations of values and behaviors. Direct similarity comparisons are less likely to be based on personal importance attributed to the behaviors or their perceived benefits. Comparisons of similarity focus on the conceptual understanding of the value or the behavior per se. Pakizeh et al. (2007) highlight the differences between these conceptual similarities and value importance judgments. In their study, participants were presented with pairs of values, and asked which value was more important to them in each pair, and they rated the importance of each value. Moreover, participants were asked to what extent the values in each pair shared a similar meaning. Then, the authors assessed whether the discrepancy in importance attributed to each pair of values was related to their perceived semantic relatedness, finding a significant association $(r=-.26, p<.001)$. Despite the modest correlation, Pakizeh et al.'s research provided the first association between importance ratings and conceptual similarity.

Therefore, the use of conceptual similarities can help to elucidate value-behavior relations. Values may have low or high conceptual similarity, while having low or high motivational force, that is, similarity and personal relevance are distinct. These differences between conceptual similarities and importance can also occur when comparing values with behavioral instantiations. This raises several questions: When we map these conceptual similarities between behavioral instantiations of values, will we obtain a structure that resembles Schwartz's value model? If not replicated, this finding would show that even the most closely related behaviors have determinants (e.g., goals, abilities) that are not isometric with the motivations underpinning values in the model (perhaps due to other factors besides values that shape behavior). This would be consistent with Bardi and Schwartz's (2003) suggestion that there may be systematic exceptions to the pattern predicted by Schwartz's model. These exceptions would occur because of the stronger value-behavior relations exhibited by some types of values (e.g., tradition, stimulation) than for other value types (e.g., benevolence, security). In contrast, if behaviors that are prototypical of values exhibit the same circular pattern, this would support the model and suggest that any departures from sinusoidal wave patterns in value-behavior relations stem largely from atypicality in the mental representation of the particular behaviors within the values. 


\section{The Present Research}

The present research assessed similarities between mental representations of values and behaviors, using similarity ratings. This approach allowed us to estimate spatial representation of behavioral instantiations alongside the spatial representation of values in Schwartz's space (see Figure 1). To develop these spatial representations, we used multidimensional scaling (MDS) to map values and behaviors. In MDS plots, items positioned more closely together can be interpreted as more similar, whereas those positioned further apart are more dissimilar (Hout et al., 2013). Thus, in addition to providing a psychological model of similarity, MDS allowed us to visualize the similarity relations between instances by reducing potentially complex similarity data to Cartesian spatial representations.

To assess the structural convergence between the behavioral instantiations of values and the values themselves, we first conducted two pilot studies to generate the behavioral exemplars and test their value representativeness. Next, we employed different methodologies to spatially map the behavioral instantiations across three empirical studies. We examined participants' perceptions of the similarities between behavioral instantiations of values with Schwartz's 10 value types (Study 1), Schwartz's four higher order values (Study 2), and other behavioral instantiations of values (Study 3).

The combined sample size across all three studies was 367 participants. This sample size enabled us to obtain relatively precise estimates of the similarity relations in each study. ${ }^{1}$ To avoid confounding cultural difference between different experiments when interpreting the behaviors (Hanel et al., 2018), participants from all three studies were British individuals living in the UK. They were recruited online via Prolific Academic (an online participant recruitment tool; https://www.prolific.co/). Each participant took part only in one study.

\section{Pilot Studies: Creating Behavioral Instantiations of Values}

In our first pilot study, 104 participants from the general public (65 women and 39 men; $M_{\text {age }}=36.14$, $\left.S D_{\text {age }}=12.63\right)$ were asked to create hypothetical situations/behaviors for each of the 10 value types from Schwartz's model, following a previously developed methodology (Hanel et al., 2018, Study 1). After being briefly introduced to the concept of human values, we asked participants to give two examples of hypothetical situations in which they considered the value types from Schwartz theory (e.g., Benevolence, Universalism, Power) to be relevant. The situations were composed of different people (participants), actions (behaviors), and places (locality). The data were then analyzed using IRAMUTEQ, free software that performs content analysis that is coded in R and Python (https://sourceforge.net/proj ects/iramuteq; Ratinaud, 2009). Through frequency analyses (e.g., Frequency of Active Words, Similarities Analysis, Word Cloud) and with the help of experts in human values, we adapted participants' most typical answers to create a list of meaningful behavioral instantiations of values. Based on the most typical answers and following the experts' suggestions, we selected the 40 best fitting behaviors (four for each of the 10 value types). Many more behaviors could have been generated and used within this research due to varied answers. However, to keep the length manageable for participants when doing the direct similarity judgments, we decided to stick with this specific number of behavioral instantiations of values.

In the second pilot study, 105 participants (51 women and $54 \mathrm{men} ; M_{\text {age }}=35.87, S D_{\text {age }}=11.85$ ) were asked to rate the extent to which the behavioral instantiations of values related to their respective value types, from $0 \%$ (not at all) to $100 \%$ (extremely). We presented the list of forty behaviors instances (four per value type). Before completing the task, participants were briefly introduced to the concept of human values. In addition to the behaviors used, nine arbitrary situations that we generated (e.g., "feeling depressed") were assigned to random value types to avoid eliciting routinely high automatic agreement with items. All but one of the behavioral instantiations of values were described as more than $50 \%$ related to their respective value types (see Table 1). The only exception was "Children eating healthy food at home," which was described as $35 \%$ related to the value type for which it was generated, conformity. Together, this validates our selection of behaviors. Also, as expected, participants indicated that the nine arbitrary behavioral instantiations of values assigned to random value types were less than $50 \%$ relevant to those values.

\section{Study I}

Suppose the behaviors obtained from our pilot research are mentally related to Schwartz's model's underlying motives and values. In that case, the spatial representations of behaviors derived from MDS should correspond with the location of the putatively relevant values in Schwartz's model. Study 1 assessed the relations between the 40 behavioral instantiations of values (see Table 1) and Schwartz's 10 value types. Although uncommon, this type of approach comparing values (and, in this case, behavioral instantiations of values) to value types is not novel in the literature. This relatively new approach to comparing values (and behavioral instantiations of values) was used by Coelho et al. (2019). They assessed whether value items could be directly compared to value types and whether these conceptual comparisons would 
Table I. Rated Degree of Relevance Between the Situations/Behaviors and the Value Types.

\begin{tabular}{|c|c|c|c|}
\hline Situations & CODE & Mean & $S D$ \\
\hline \multicolumn{4}{|l|}{ Benevolence $(\omega=.56)$} \\
\hline Nurses taking care of patients in hospital & BEI & 80.60 & 15.30 \\
\hline Mothers looking after their children at home & BE2 & 80.60 & 20.68 \\
\hline Volunteers providing food for homeless people in the community & BE3 & 77.68 & 20.95 \\
\hline Workers helping each other at workplace & BE4 & 74.01 & 17.79 \\
\hline \multicolumn{4}{|l|}{ Universalism $(\omega=.58)$} \\
\hline Environmentalists planting new trees in the forest & UNI & 80.82 & $|8.5|$ \\
\hline Social workers helping people in their local communities & UN2 & 75.38 & 18.20 \\
\hline Zookeepers taking care of animals & UN3 & 71.55 & 20.73 \\
\hline Teachers helping students at school & UN4 & 60.66 & 25.25 \\
\hline \multicolumn{4}{|l|}{ Self-direction $(\omega=.59)$} \\
\hline Artists creating a new painting design & SDI & 83.42 & 13.77 \\
\hline Authors writing a new book & SD2 & 81.15 & 15.19 \\
\hline Children drawing a picture at home & SD3 & 76.70 & 17.40 \\
\hline Students learning at school & SD4 & 57.21 & 24.96 \\
\hline \multicolumn{4}{|l|}{ Stimulation $(\omega=.65)$} \\
\hline Skydivers jumping from a plane & STI & 91.24 & 11.14 \\
\hline Adventurers climbing a mountain & ST2 & 86.21 & 14.79 \\
\hline Children playing at the park & ST3 & 72.26 & 21.02 \\
\hline Athletes running on a track & ST4 & 67.22 & 21.30 \\
\hline \multicolumn{4}{|l|}{ Hedonism $(\omega=.75)$} \\
\hline People going to a club or beach & HEI & 73.78 & 22.95 \\
\hline Gamers playing at home & HE2 & 72.28 & 23.43 \\
\hline Teenagers having a drink in a pub & HE3 & 68.50 & 26.38 \\
\hline People eating at a restaurant & HE4 & 64.71 & 22.64 \\
\hline \multicolumn{4}{|l|}{ Achievement $(\omega=.64)$} \\
\hline Athletes winning the Olympics & $\mathrm{ACl}$ & 90.01 & 13.33 \\
\hline Students graduating from university & $A C 2$ & 87.56 & 12.69 \\
\hline Employees getting a promotion at work & $\mathrm{AC} 3$ & 84.28 & 13.95 \\
\hline Teachers accomplishing their duties at school & AC4 & 71.61 & 18.19 \\
\hline \multicolumn{4}{|l|}{ Power $(\omega=.65)$} \\
\hline Prime-ministers or presidents making decisions at parliament & POI & 84.55 & 15.87 \\
\hline Politicians giving speeches in town halls & $\mathrm{PO} 2$ & 72.30 & 18.82 \\
\hline Managers chairing a meeting at the workplace & $\mathrm{PO} 3$ & 69.04 & 20.35 \\
\hline Teachers disciplining students at school & $\mathrm{PO} 4$ & 67.26 & 23.17 \\
\hline \multicolumn{4}{|l|}{ Security $(\omega=.73)$} \\
\hline Police officers arresting criminals in the streets & SEI & 85.47 & 13.11 \\
\hline Police officers patrolling the streets & SE2 & 84.94 & 13.43 \\
\hline Parents taking care of their children at home & SE3 & 78.68 & 19.10 \\
\hline Security guards locking doors in a bank & SE4 & 75.28 & 20.92 \\
\hline \multicolumn{4}{|l|}{ Tradition $(\omega=.64)$} \\
\hline Couples getting married at church & TRI & 82.97 & 17.22 \\
\hline Religious people praying at home & TR2 & 81.42 & 17.03 \\
\hline Priests giving sermons at church & TR3 & 79.99 & 19.41 \\
\hline Individuals visiting family at home & TR4 & 67.64 & 21.93 \\
\hline \multicolumn{4}{|l|}{ Conformity $(\omega=.6 \mathrm{I})$} \\
\hline Students following a dress-code at school & $\mathrm{COI}$ & 73.88 & 22.67 \\
\hline Prisoners following prison rules & $\mathrm{CO} 2$ & 72.89 & 25.64 \\
\hline Workers respecting colleagues & $\mathrm{CO} 3$ & 65.49 & 24.68 \\
\hline Children eating healthy food at home & $\mathrm{CO} 4$ & 34.93 & 28.53 \\
\hline
\end{tabular}

Note: $\omega=$ McDonald's omega.

replicate Schwartz's structure through conceptual representations of values. Their findings provided support to the value model. Our study tested whether the spatial representation for behavioral instantiations of values replicates Schwartz's (1992) value space. Behaviors that were judged as similar to one value are near each other in the plot.

\section{Method}

Participants. One hundred twenty-five participants completed an online survey. Two participants failed the instructional manipulation check (IMC) twice (Oppenheimer et al., 2009) and/or two test items spread across the main task and were excluded. Of 
the remaining 123 participants, 54 were men (43.9\%) and 69 women $(56.1 \%)$, with a mean age of 38.93 $(S D=11.32)$.

Material and Procedure. Participants were briefly introduced to human values. Next, they were asked to rate the similarity between 40 instantiations (e.g., Adventurers climbing a mountain; Police officers patrolling the streets) and Schwartz's (1992) 10 value types (e.g., universalism, hedonism). Participants rated the similarity of each pair using a slider scale from $0 \%$ (not at all) to $100 \%$ (extremely). To avoid boredom and fatigue, participants only rated 200 out of the 400 possible comparisons. These 200 pairs were randomly assigned to the participants. This way, all pairs were rated a similar number of times across participants.

\section{Results and Discussion}

MDS Methodology. First, we calculated the mean similarity ratings of all 400 comparisons. Next, we performed an ordinal MDS using the PROXSCAL algorithm in SPSS. This algorithm creates a geometric representation of the similarity data in terms of the proximity between the items (Hout et al., 2013). We selected the Torgerson configuration (also known as classical MDS) as the initial configuration, creating a two-dimensional representation of high-dimensional data (Brandes \& Pich, 2007). We used Stress-I to assess the model fit (Jaworska \& ChupetlovskaAnastasova, 2009), following Sturrock and Rocha's (2000) suggested cut-offs. Lower values indicate a better model fit. For this study, when 40 behaviors are spread across a two-dimensional plane, a cut-off lower than .35 is recommended. The results indicate a good model fit (Stress-I $=.13$ ).

We assessed how closely this spatial representation of behaviors aligned with the representation of values in Schwartz's model, using $\mathrm{R}$ software ( $\mathrm{R}$ Development Core Team, 2015). To assess this fit, we used Procrustes analysis ("Protest"; Peres-Neto \& Jackson, 2001), which tests the degree to which two sets of points align. Specifically, Protest compares two ordinations using symmetric Procrustes analysis (Oksanen, 2015) by minimizing the sumof-squared differences through rescaling the configurations to a common size, mirror reflecting (if necessary), and rotating (Peres-Neto \& Jackson, 2001). Protest is also known as an analysis of congruence (Oksanen, 2015). In other words, using Protest in our studies enabled us to assess the alignment of the behavioral spatial arrangements in our data with the theoretical spatial arrangement of values from Schwartz's model. The "coefficient of agreement" between the data and the hypothetical structure of Schwartz's model is quantified by an effect size similar to a correlation coefficient, which can be labeled $r_{\mathrm{m}}$ (as used in Coelho et al., 2019) and by whether it is statistically significant (Oksanen, 2015). The larger the $r_{\mathrm{m}}$, the better the fit. If $r_{m}$ is statistically significant (i.e., $p<.05$ ), this implies agreement between the data and hypothetical structure. We specified Schwartz's model (i.e., the target structure) by creating a hypothetical ideal structure with the four higher order value types placed along a circle, $90^{\circ}$ apart from each other. For example, self-enhancement values were initially placed at the bottom, $x=0, y=-0.5$. In contrast, self-transcendence values were expected to be placed at the top, $x=0, y=0.5$ (the R-code can be found in the online Supplementary Materials, see the file "Protest analsis.R"). Another caveat is that we measured agreement by a high standard, because the dimensions were treated as orthogonal and the instances perfectly positioned in relation to the dimension. A more flexible approach may have shown higher fit. Nonetheless, our approach enabled a consistent test in line with a perfect circular model. The results indicated a good match between the spatial representations of instantiations and the ideal structure of Schwartz's model $\left(r_{\mathrm{m}}=.79, p<.001\right)$.

We used the convex hull (dashed lines connecting the behaviors) to facilitate data interpretation by providing the smallest convex set of behaviors for each higher order value from Schwartz's model. As shown in Figure 2, most behavioral instantiations of values from the same higher order value were clustered together. In addition, the putatively opposing behaviors (based on the values from which they were derived) were in opposite positions on their respective dimensions, easily seen in the conservation versus openness to change dimensions. However, the spread of the behaviors derived from selfenhancement values is large. Some of them visually oppose each other in the spatial set (AC1-PO4), rather than being adjacent as in Schwartz's (1992) model. Also, some behaviors derived from selftranscendence values were mixed with conservation behaviors, including "Teachers helping students at school" (UN4) and "Mothers looking after their children at home" (BE2).

In sum, most of the behavioral instantiations were clustered with behaviors from the same value type, indicating that behaviors derived from values mirror the structure of the values when directly compared to them. However, the abstractness of values and the potential for behaviors to express different values at once (Bardi \& Schwartz, 2003; Schwartz, 2013) also led us to expect some deviations. The deviations that we observed illustrate this. For example, "Teachers helping students at school" was generated in response to a self-transcendence value type (Universalism; UNI04). However, the content of the value can also imply, for instance, conservation motives because it involves teachers doing their jobs. This dual relevance might help to explain why this specific behavior mixed with conservation across the two-dimensional space. The varied instances of intermixing between 


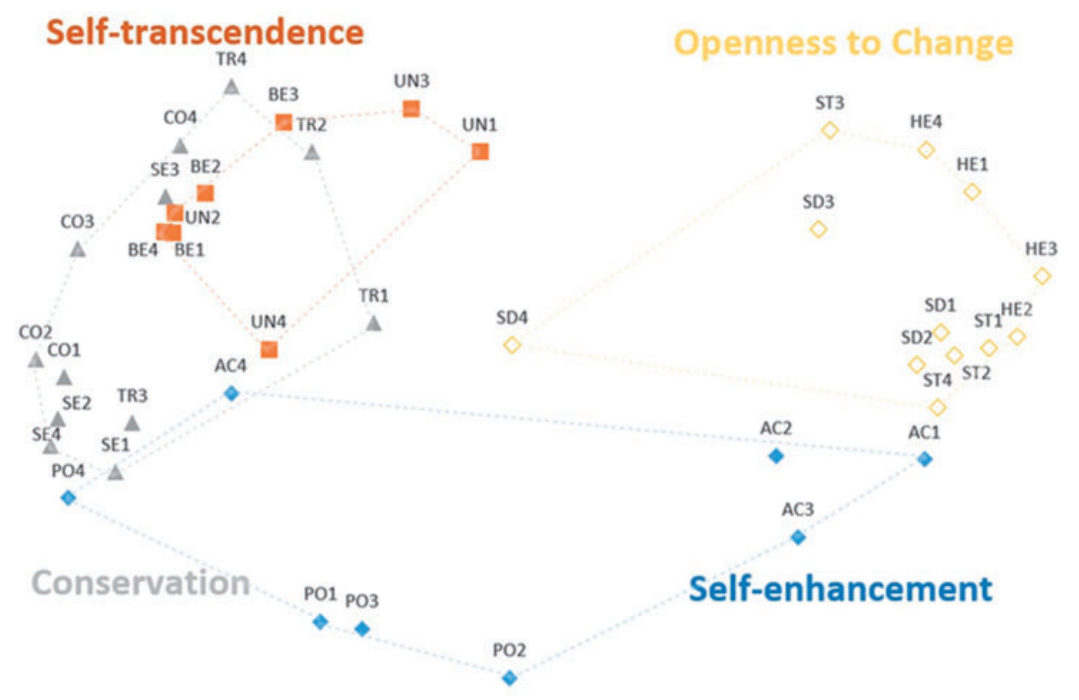

Figure 2. MDS Structure for Similarities Ratings Between Behaviors and Value Types (Study I). Convex hull: dashed lines connecting behavior groups. AC: Achievement, BE: Benevolence, CO: Conformity, HE: Hedonism, PO: Power, SD: Self-direction, SE: Security, ST: Stimulation, TR: Tradition, UN: Universalism. ${ }^{2}$

the behaviors and other values show that even the behaviors generated to represent particular values can represent other value types.

\section{Study 2}

In Study 2, we asked participants to place the behaviors directly along Schwartz's two dimensions, selfenhancement versus self-transcendence and openness versus conservation. Behavioral instantiations of values placed closer to one end of the dimension indicated that it is more characteristic of this end. This method is helpful because the two dimensions are essential core features of the model, reflecting the contrasting motives. In addition, the method is a more direct assessment of the value space in Schwartz's mode insofar as it directly plots participants' responses on the two dimensions, rather than indirectly inferring their positions on these dimensions via MDS using similarity ratings.

\section{Method}

Participants. Participants were 113 individuals, including 65 women $(57.5 \%)$ and 48 men $(42.5 \%)$, with a mean age of $37.48(S D=12.21)$. No participant failed the IMC (Oppenheimer et al., 2009) and/or test items.

Material and Procedure. Before completing the task, participants were presented with a one-paragraph summary of Schwartz's (1992) theory. This summary explained how the two value dimensions are specified. Next, participants were asked to position the behaviors between opposing ends on each of his two dimensions. Participants used a 9-point bipolar scale, with the opposing higher order value domains identified at each end. Behaviors that participants placed closer to one end should be more representative of that end of the dimension. If they judged the behavior as not representative of either end of a dimension, they could select the middle point of the scale.

\section{Results and Discussion}

The spatial representation (Figure 3) was plotted from the means of the behavioral instantiations for both dimensions, with self-enhancement versus selftranscendence on the $x$-axis and openness to change versus conservation on the $y$-axis. This method allows the coordinates of behavioral instantiations in the model to be directly checked without needing any optimization of fit. Behavioral instantiations of values originating from self-enhancement values should be on the left of the $x$-axis, while those originated from self-transcendence values should be on the right. Similarly, the openness to change behaviors should appear at the top of the $y$-axis, with conservation behaviors at the bottom.

The results can be seen in Figure 3. First, five behaviors (AC1, AC2, AC3, PO2, and PO3) promoting self-enhancement values were more likely to occur on the self-enhancement end of the self-enhancementto-self-transcendence value dimension than on the other end, which comprised all the behaviors promoting self-transcendence values. Second, most of the behaviors promoting conservation values were more likely to occur on the conservation end of the conservation-to-openness dimension than on the other end, which comprised most behaviors promoting openness values.

However, the positions of the behaviors only partly resembled Schwartz's model, despite the significant match to Schwartz's hypothetical configuration $\left(r_{\mathrm{m}}=.71, p<.001\right)$. All the openness behaviors were strongly associated with the self-enhancement pole, with associations even higher than the self- 


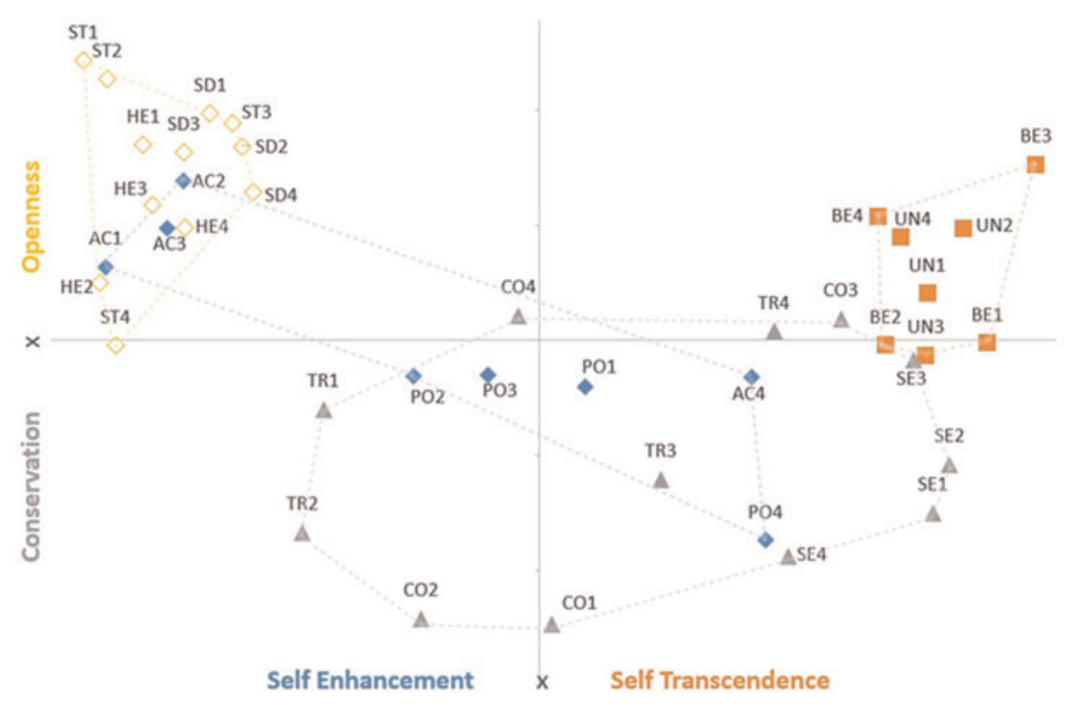

Figure 3. Behaviors Placed Among Schwartz's Dimensions (Study 2). ${ }^{3}$

enhancement behaviors themselves. These higher associations to an adjacent pole also happened with most of the self-transcendence behaviors, which were associated with the openness pole. Within the circumplex model, their behaviors are expected to be near their original value types. However, stronger association to the adjacent value types instead of the original values from which they were derived indicates that they have multiple underlying motivations. Thus, while from Schwartz's theoretical perspective, the two dimensions are orthogonal, the results indicate that behavioral instantiations of values of openness and self-transcendence both express putatively unrelated value-motives.

The deviations of the behavioral instantiations of values from the model included six behaviors positioned at the opposite end of the dimension containing the values from which the behaviors were derived. Three behaviors from the self-enhancement end were positioned slightly toward self-transcendence, and three conservation behaviors were positioned toward openness to change. Thus, individuals might think of the behaviors through different motivations. For example, "Teachers disciplining students at school" (PO4) was derived as an example of selfenhancement values. However, one can interpret this behavior as supporting a self-transcendence motivation, seeing this discipline as something that aims to help the students, instead of seeing it as a demonstration of power or authority from the teacher. Another example is "Children eating healthy food at home" (CO4), which was not well related to its respective value type, conservation, in Study 2. Despite the derivation of this behavior from conservation, one can also think of this behavior as an opportunity to try new and alternative types of food, thereby pursuing openness to experience. Other similar instances of behaviors near alternative values were "Prime-ministers or presidents making decisions at parliament"
(PO1), "Teachers accomplishing their duties at school" (AC4), "Workers respecting colleagues" (CO3), and "Individuals visiting family at home" (TR4). These instances demonstrate the inherent links between many behaviors and multiple values, even when focusing on behaviors that are judged (in our pilot samples) as typical of a particular value.

\section{Study 3}

The aim of Study 3 was to examine the relations among behaviors through similarity judgments between pairs of behaviors. Comparing behaviors with other behaviors allows a direct assessment of whether they result in the same motivational space as values, even without participants' explicit considerations of values.

\section{Method}

Participants. Participants were 135 individuals. Four participants were excluded because they failed the IMC twice (Oppenheimer et al., 2009) and/or two test items. Thus, the final sample consisted of 131 participants with a mean age of $39.48(S D=11.43)$, including 71 women $(54.2 \%)$ and 60 men $(45.8 \%)$.

Material and Procedure. Participants were asked to rate the extent to which pairs of the 40 behaviors are similar to one another, using a slider scale ranging from $0 \%$ (not at all) to $100 \%$ (extremely). An example of the task can be seen in the Supplementary Material (OSM.docx). Because the total number of pairs was large (780), each participant did only a randomly selected one-third of them (260).

\section{Results and Discussion}

An ordinal MDS was performed using the PROXSCAL algorithm and Torgerson configuration. 
Once again, the results indicated a good model fit, Stress- $\mathrm{I}=.23$, which is lower than the recommended level of .35 (Sturrock \& Rocha, 2000). The behavioral structure (Figure 4) aligned with Schwartz's model $\left(r_{\mathrm{m}}=.59, p<.001\right)$.

The behaviors exhibited some congruence with Schwartz's spatial representation of values. For instance, openness to change and conservation (i.e., the gray triangles and yellow diamonds) were at opposite sides of the space. However, the behaviors were not always consistent with the model. In particular, self-transcendence behaviors were mixed with both conservation and self-enhancement in a position near the middle of the plane. This mixing may have occurred because these two higher order values have a social focus, representing how individuals socially relate to and affect others (e.g., Schwartz et al., 2012). Despite this deviation, the self-transcendence behaviors were more clustered than the other three higher order behavioral instantiations of values, indicating a greater similarity between behaviors in this value domain.

There are viable alternative explanations for the obtained behavior mapping - in addition to the motivations encompassed within Schwartz's model. Consistent with the self-other distinction in Schwartz's model, most of the behaviors that are positioned at the right of the spatial representation showed a higher focus on personal gains (e.g., "People eating at a restaurant," "Teenagers having a drink in a pub"), whereas the behaviors more to the left of the space were more social in focus (e.g., "police officers arresting criminals," "zookeepers taking care of animals"). The behaviors may also have differed in familiarity to the participants. The behaviors on the left refer to specific roles not necessarily performed by the participants ("Security guards locking doors in a bank," "prime-ministers making decisions"). In contrast, the ones from the right are more likely to be performed frequently ("Gamers playing at home," "People going to a club or beach"). These differences in focus and familiarity across the space may explain these differences in alignment between the behaviors and the values from which they were derived.

In summary, when making direct comparisons between value-derived behaviors without explicitly invoking values, the data again broadly aligned with Schwartz's model, although the fit was not as strong as in our Studies 1 and 2, in which behaviors were compared with values. Although openness to change and conservation behaviors were in opposing ends of the space, self-transcendence behaviors were in the center, mixed with conservation and selfenhancement behaviors. The mental representation of these behaviors may be affected by their focus (social or personal) or their familiarity, among other factors.

\section{General Discussion}

Across three studies, we compared spatial representations of value-related behaviors that were explicitly derived from people's mental representations of the values via similarity judgment to the spatial positions of values in Schwartz's model of human values. The key purpose of this research was to assess whether similarly derived spatial representations of valuerelated behaviors are in a common space with the values themselves, both accounted for by Schwartz's model. We therefore assessed the relations between human values and behavioral instantiations of values through various tasks using direct judgments

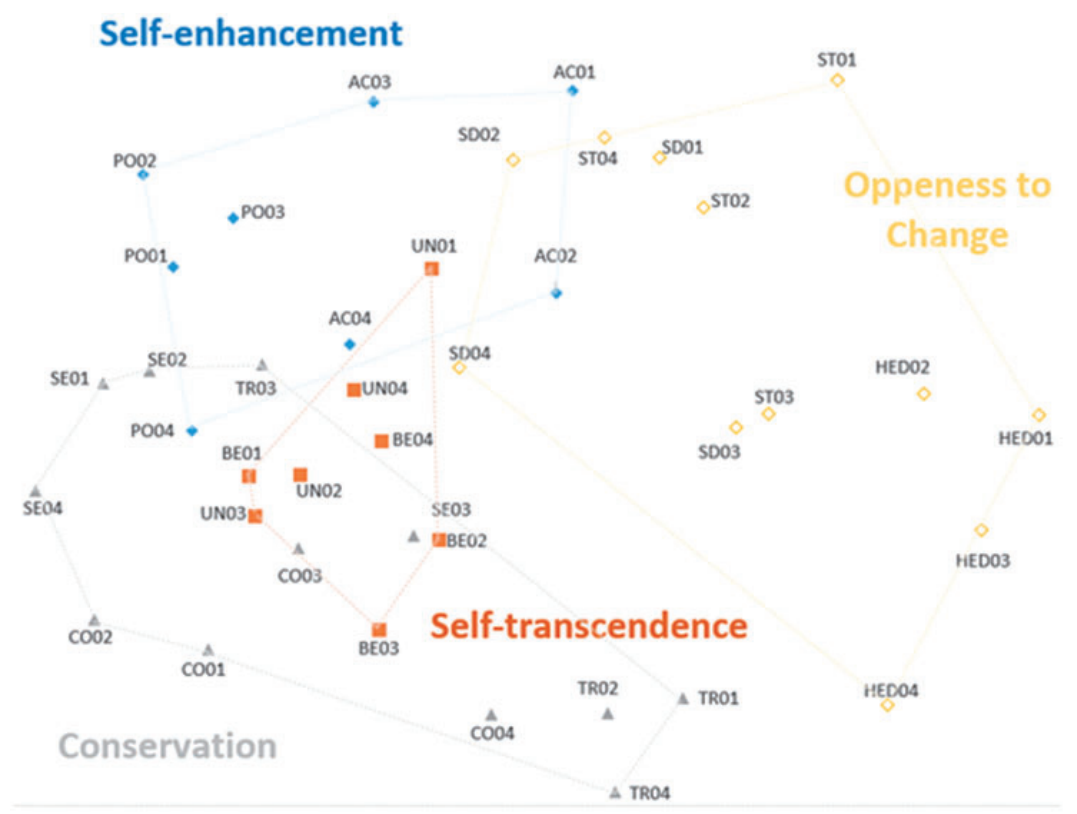

Figure 4. MDS Spatial Representation of Behavior Similarities (Study 3). ${ }^{4}$ 
of conceptual similarities. These tasks provided the first spatial representations that capture how people interpret and understand behaviors in relation to values. The tasks also enabled us to evaluate whether these mental representations of the behaviors are spatially represented in a way that aligns the spatial positions of values in Schwartz's model, as these behaviors putatively expressed the motivations underpinning the values. We assessed whether the mental representations of the behaviors are consistent with the values and motivation dimensions in Schwartz's model, as the behaviors originated from the same values.

\section{Mapping Behavioral Instantiations}

Studies 1 and 2 made direct comparisons between values and behaviors using different methods. In Study 1, participants rated the similarities between behaviors and all 10 lower order value types in Schwartz's model. The resulting spatial representation showed substantial congruence with Schwartz's model, with most behaviors clustered together with behaviors from the same higher order value. Also, the map visually reproduced aspects of motivational agreement versus conflict, with conservation opposing openness to change behaviors and self-enhancement opposing self-transcendence. Nevertheless, it is important to highlight that selfenhancement behaviors were widely spread across the two-dimensional space, indicating more variation in the way individuals interpret these behaviors, associating them with other value types. Another interesting finding is that behaviors derived from self-transcendence values were mixed with behaviors derived from conservation values. This mixing might have occurred because of the social focus of these two higher order values (e.g., Schwartz et al., 2012). For instance, consider the behavior "Nurses taking care of patients in hospital" (BE1). The underlying motivation is benevolent, because the behavior represents professionals taking care of other people. However, it can also be interpreted as individuals trying to maintain stability and security and improve people's health, which taps into conservation motivations.

The spatial representation from Study 2 also showed substantial agreement with Schwartz's model. One interesting finding is that both openness to change and self-transcendence behaviors were highly clustered, indicating strong congruence between their respective behavioral instantiations of values. In contrast, conservation and selfenhancement behaviors were more heterogenous and were spread across the plane, and even sometimes occur at the opposite end of the space. One possible explanation relies on the motivations people attribute to these behaviors. For example, consider the behavior "Prime-ministers making decisions at parliament" (PO1), which was rated in Study 1 as a good example of power. This behavior can also be considered to express concerns for the welfare of others and therefore is an alternative example of self-transcendence behavior.

The multiple motivations potentially underlying the behaviors made it relevant to consider the structure revealed by comparing the value-instantiating behaviors to one another, without any direct reference to values. In Study 3, we removed the explicit influence of values, and performed direct comparisons of similarity between all 40 behaviors. It was an open question whether this would generate a new spatial representation or replicate Schwartz's structure. The results showed some congruence with Schwartz's model and some differences. Conservation and openness to change behaviors were at opposite sides of the spatial representation, agreeing with the model. However, self-transcendence behaviors were positioned close to the center of the spatial representation, mixing with self-enhancement and conservation behaviors, so not fully agreeing with the model. This, again, can be understood when assessing the content of these studies. For instance, "Teachers helping students at school" (UN4) and "Teachers accomplishing their duties at school" (AC4), which are behaviors from opposite sides in the selfenhancement versus self-transcendence dimension. While universalism represents the "understanding, appreciation, tolerance, and protection for the welfare of all people and for nature" (Schwartz, 1992, p. 12), achievement is characterized by "personal success through demonstrating competence according to social standards" (Schwartz, 1992, p. 8). However, despite their implied motivational differences from Schwarz's model, the behaviors are closely related. Therefore, although there were notable deviations from Schwartz's value model, the behavioral instantiations were broadly consistent with it.

However, Study 3 also revealed the importance of considering diverse ways in which behaviors vary. The behaviors more to the left of the spatial representations corresponded to a higher social focus and were less frequent examples of daily behaviors. On the other hand, the behaviors more to the right of the spatial representation had a more personal focus and are more typical of daily life. Given the way our pilot study generated these behaviors, the reasons for this difference may be a natural consequence of how people mentally construct the behaviors relevant to the values.

Furthermore, we discuss why certain behaviors consistently deviated across studies from other behaviors initially thought to express the same value. For instance, consider the behavior "Teachers disciplining students at school" (PO4). This behavior originated from power and was considered a good example of that value type in our pilot studies. However, this behavior was positioned among conservation behaviors (Studies 1 and 3) and positioned on the self- 
enhancement side close to conservation (Study 2). We believe that this deviance arose because the behavior can be understood in different ways. For example, disciplining can be easily interpreted as a demonstration of power, as it shows authority over individuals. Within a specific context, such as school, however, disciplining can be understood as an example of order or self-restraint, which are conservation values. Therefore, such deviations across studies further demonstrate the importance of assessing the motivation of behavior across methods. One behavior or instantiation can be directly extracted from a value, be considered a good example and still be more associated with behaviors derived from other values, suggesting that the underlying motivation of behaviors can vary.

One interesting additional finding is that the fit of the behaviors to the structure of Schwartz's model varied across studies. When participants compared the behaviors directly with the 10 value types, the resulting fit with Schwartz's model was highest (Study $1 ; r_{\mathrm{m}}=.79$ ). When the behaviors were compared with the higher order dimensions, the fit was lower (Study 2; $r_{\mathrm{m}}=.71$ ). Finally, when behaviors were compared among each other, the fit was lowest (Study 3; $r_{\mathrm{m}}=.59$ ). Thus, even though all three models significantly congruent with Schwartz's model, there were differences in the degree of agreement.

This raises the questions on whether the mental representation of value instantiations in relation to the lower order value domains better isolates the motivational relations predicted by Schwartz's model than does the mental representation of value instantiations in relation the higher order domains or specific behaviors. Comparison to the lower order value types may allow participants to differentiate between the underlying motivation of each behavior more clearly. For example, in Study 1, participants contrasted the behavior with more specific value types such as benevolence, which was described in the task as "Preservation and enhancement of the welfare of people with whom one is in frequent personal contact." In contrast, in Study 2, participants contrasted behaviors with higher order value types such as self-transcendence, which is described in the task as concern "with enhancement of others and transcendence of selfish interests" (Schwartz, 1992, p. 15). Finally, when behaviors are compared among each other, as done in Study 3, the many underlying motivations of each behavior may result in a noisier outcome because people might attribute different underlying mechanisms to each behavior (cf. Hanel et al., 2018). Comparison to lower order value types may be the optimal level of analysis for pulling out the motivational conflicts suggested by Schwartz's model, since they present more specific core characteristics, enabling individuals to distinguish better between slightly different underlying motivations of similar behaviors. Of course, one relevant caveat is that we applied a visual method of generating the mental representations of value instantiations in relation to the higher order values in Study 2 (instead of using MDS), and it would be worthwhile for future research to check whether an MDS format also yields lower fit to the higher order types than to the lower order types.

Finally, to provide further support for our findings, we visualized how the data from our studies are structured using heatmaps (see .xlsx files) and cluster analyses (see Cluster Analysis.docx). Both approaches replicated the findings we obtained using MDS. The behaviors that were closer in the two-dimensional plots presented above revealed higher degrees of similarities in the heatmaps and were grouped in the cluster analyses.

\section{Is Schwartz's Structure Replicated?}

The findings helped to reveal many of the subtle complexities that should be considered when tackling questions about the role of values in human social cognition and behavior. In the cognitive literature, conceptual categorization is frequently interpreted to occur based on the features that compose the concepts, grouping new items to similar old ones (e.g., Hahn \& Chater, 1997). This process can happen in different ways because these classifications are based on personal experiences and interpretations. For example, some people might classify different animals, such as cats, tigers, dogs, and wolves, as types of felines and canines, while others might classify them as pets and wild animals. By comparison, values are relatively abstract concepts and more fluid in construal, and these complexities appear when using conceptual categorizations of behavioral instantiations that were directly originated from values. In light of this fluidity, it is remarkable that people can map these abstract concepts in systematic ways that reflect extant assumptions about their motivational relations. In other words, despite the tremendous range of behaviors that may relate to values, there are core characteristics that enable people to categorize them in ways that reflect their putative motivational congruences and incongruences. That we observed various departures from this alignment with Schwartz's model neither refutes the circular model of values nor the feasibility of linking behaviors to values. The results of our research suggest that behavioral instantiations of values are represented in a similar way to the values themselves. If people were not taking the behaviors' underlying motivations into account, a behavioral spatial representation that aligns with Schwartz's value model would seem unlikely. 


\section{Limitations}

One key consideration is the relation between similarity judgments and importance ratings. To judge the importance of different objects or assess their similarities, people operate under assumptions of what these judgments represent. Therefore, the similarity judgments depend on individual knowledge, which may relate very differently to judgments of importance. Imagine, for instance, that an individual, disappointed with their religion, decided to abandon it. This change is likely to lower their endorsement of tradition values. However, when presented with similarity judgments between these values and behaviors such as "Couples getting married at church" (TR1) and "Priests giving sermons at church" (TR3), it is likely that they will attribute a high similarity, because of the content of these values and behaviors, but this is likely to be independent of their value endorsements (i.e., whether tradition is important or not). Hence, the exact nature of the relations between similarity judgments and importance ratings still begs further analysis. Prior research (Maio \& Olson, 1998) has suggested that the cognitive underpinning of values is weak, which could raise questions about whether the structures found in our studies, using similarity judgments, are related to value importance in a systematic manner. Suppose a participant rates the value of equality as very important to them. In that case, they might want to rate broad-mindedness or social justice as very important, because they are similar at the system level, and they would like to appear consistent. However, the instantiations of equality that the participant brings to mind may or may not suit this convergence in importance ratings, depending on whether or not the instantiations support these additional values. This interplay between value importance and the role of value instantiations is an important additional avenue for future research.

Moreover, another question that can be raised concerns the number of participants in our studies, ranging from 113 to 131 . Although a higher number is desired, the samples were restricted to these numbers because of limited resources. However, the fact that the findings are overall consistent across studies indicates that the sample sizes were sufficient. Also, the stress values across our studies suggest that the samples were large enough for the MDS to find a spatial solution that represented the data well.

\section{Future Studies}

Our research is only the first step in rigorously mapping the connections of behavioral instantiations of values to the putatively relevant values. There are cross-cultural differences in how people endorse and instantiate different values (e.g., Coelho et al., 2019; Hanel, 2016; Hanel et al., 2018). Future research needs to consider the impact of differences between behaviors generated as exemplars of distinct values in different cultures. This should include applying the methods we have used here in different nations and cataloguing different attributes of behaviors (e.g., actor, context, time) for different values. Such efforts will help to assess the extent to which the alignments and departures from Schwartz's model represent systematic, universal variations or context-specific and behavior-specific effects. However, given the extensive cross-cultural similarities in value importance ratings (Hanel et al., 2019; Schwartz \& Bardi, 2001), value structure (Schwartz, 1992; Schwartz \& Sagiv, 1995; Schwartz et al., 2012), and some similarities in value instantiations (Hanel et al., 2018), we expect that our findings will be replicated cross-culturally.

Moreover, another possible direction for future studies is to begin modeling vital individual differences in mental representations of values. For instance, one person might interpret a medic's daily actions as associated with self-transcendence motivations because of the intentions to help other individuals. However, some people may interpret these actions as self-enhancement motivated, as a way to improve the medic's professional skills or to get more money from the patients. This difference in a general benevolent system of behavior attribution versus selfenhancing system of behavior attribution may alter how values are conceived and the behaviors that are brought to bear on the values.

It would also be useful for future research to consider whether importance judgments influence similarity judgments. To make comparisons regarding the similarities of items, people are subject to a cognitive understanding of the items. They might also be influenced by their subjective evaluations of the personal significance or importance of the items, particularly in the case of values and relevant behaviors. However, the exact nature of the relations between similarity judgments and importance ratings is still only partly clarified. In prior research, Pakizeh et al. (2007) attempted to assess how semantic relatedness and importance ratings of values were related to each other. They found a significant, but modest, association $(r=-.26, p<.001)$. If importance had a major influence on similarities judgments, we would expect higher associations between them. Nevertheless, the interaction between these components remains a matter for future study.

Finally, the approach used in our research - asking individuals of a given culture to list typical behaviors of a set of values - can also elicit typical behaviors that threaten a value. For example, one individual to whom freedom is very important may see the need to stay at home during lockdown restrictions as a threat to this value. Thus, these other types of instantiations could be assessed in future research, attempting to visualize whether these value threatening behaviors might also present similar associations 
(although, likely to be negative) to the motivational circle of values.

\section{Conclusion}

In summary, our findings help to address a frequent problem in research on value-behavior relations. Most studies simply assume a priori that a behavior has a connection to a value or set of values. There is rarely any critical examination of the actual degree of agreement that individuals have in their mental representations of values and the behavioral instantiations of them. Our findings illustrate a method for identifying which behaviors align well with the values from which they were methodologically derived and which behaviors align poorly. At the same time, the results illustrate the need to consider other factors that may cause values and behavioral instantiations of values to align with different motivations, including other variables in behavioral categorization (e.g., frequency, personal relevance) and individual differences. These insights may help researchers select value-relevant behaviors in future studies of value-behavior relations by carefully considering the values' mental representations.

\section{Data accessibility statement}

All data (.sav files), statistical code (.sps and .R files), heatmaps (.xlsx files), Cluster Analysis (Cluster Analysis.docx), and Online Supplementary Material (OSM.docx) are available through the link https://osf.io/zasq3/?view_on$1 \mathrm{y}=75 \mathrm{f} 1644192 \mathrm{~d} 14703 \mathrm{bbae} 843 \mathrm{~d} 30 \mathrm{e} 3 \mathrm{ec} 6 \mathrm{~b}$. Within the OSM file, we provide task instructions and examples, Sheppard Plots and Stress-per-point tables.

\section{Declaration of Conflicting Interests}

The author(s) declared no potential conflicts of interest with respect to the research, authorship, and/or publication of this article.

\section{Funding}

The author(s) disclosed receipt of the following financial support for the research, authorship, and/or publication of this article: This study received financial support from the Coordination for the Improvement of Higher Education Personnel (CAPES Foundation, Brazil; Proc. 99999.013718/ 2013-04 to G. L. D. H. C.), and from the School of Psychology, Cardiff University (psych.cf.ac.uk) and the Economic and Social Research Council (ESRC; www.esrc. ac.uk) to P. H. P. H. (ES/J500197/1). The funders had no role in study design, data collection and analysis, decision to publish, or preparation of the manuscript. The studies reported here and in the Supplemental Materials, alongside parts of the Introduction and General Discussion, are described in the PhD thesis of the first author (Coelho, 2018).

\section{ORCID iDs}

Gabriel Lins De Holanda Coelho (D) https://orcid.org/00000003-4744-3151

Paul H. P. Hanel (D) https://orcid.org/0000-0002-3225-1395

\section{Notes}

1. For example, in Study 2, 113 participants judged the similarity between instantiations and values on a 9-point bipolar scale (see Study 2 for details). Assuming an average standard deviation of 1.88 for the 9-point scale (as found by Coelho et al., 2019 in a methodological similar study), the $95 \%$ confidence interval across each similarity judgment would be on average $[M-1.96 \times 1.88 / \operatorname{sqrt}(113), \quad M+1.96 \times 1.88 /$ $\operatorname{sqrt}(113)]=[M-0.35, M+0.35]$. In other words, the width of the confidence intervals would be only $0.35+0.35=0.70$ points on our 9-point scale, which is fairly narrow.

2-4. Athletes winning the Olympics, AC1; Students graduating from university, AC2; Employees getting a promotion at work, AC3; Teachers accomplishing their duties at school, AC4; Nurses taking care of patients in hospital, BE1; Mothers looking after their children at home, BE2; Volunteers providing food for homeless people in the community, BE3; Workers helping each other at workplace, BE4; Students following a dresscode at school, CO1; Prisoners following prison rules, $\mathrm{CO} 2$; Workers respecting colleagues, CO3; Children eating healthy food at home, $\mathrm{CO} 4$; People going to a club or beach, HE1; Gamers playing at home, HE2; Teenagers having a drink in a pub, HE3; People eating at a restaurant, HE4; Prime-ministers or presidents making decisions at parliament, PO1; Politicians giving speeches in town halls, PO2; Managers chairing a meeting at the workplace, PO3; Teachers disciplining students at school, PO4; Artists creating a new painting design, SD1; Authors writing a new book, SD2; Children drawing a picture at home, SD3; Students learning at school, SD4; Police officers arresting criminals in the streets, SE1; Police officers patrolling the streets, SE2; Parents taking care of their children at home, SE3; Security guards locking doors in a bank, SE4; Skydivers jumping from a plane, ST1; Adventurers climbing a mountain, ST2; Children playing at the park, ST3; Athletes running on a track, ST4; Couples getting married at church, TR1; Religious people praying at home, TR2; Priests giving sermons at church, TR3; Individuals visiting family at home, TR4; Environmentalists planting new trees in the forest, UN1; Social workers helping people in their local communities, UN2; Zookeepers taking care of animals, UN3; Teachers helping students at school, UN4.

\section{References}

Aavik, T., \& Dobewall, H. (2017). Where is the location of "health" in the human values system? Evidence from Estonia. Social Indicators Research, 131(3), 1145-1162. https://doi.org/10.1007/s11205-016-1287-4

Adams, R. (2014). Schools 'must actively promote British values'. The Guardian. http://www.theguardian.com/edu cation/2014/nov/27/schools-england-promote-britishvalues

Bardi, A., \& Schwartz, S. H. (2003). Values and behavior: Strength and structure of relations. Personality \& Social Psychology Bulletin, 29(10), 1207-1220. https://doi.org/ 10.1177/0146167203254602 
Boer, D., \& Fischer, R. (2013). How and when do personal values guide our attitudes and sociality? Explaining cross-cultural variability in attitude-value linkages. Psychological Bulletin, 139(5), 1113-1147. https://doi. org/10.1037/a0031347

Brandes, U., \& Pich, C. (2007). Eigensolver methods for progressive multidimensional scaling of large data. In M. Kaufmann \& D. Wagner (Eds.), Graph drawing (pp. 42-53). Springer Berlin Heidelberg. http://link. springer.com/chapter/10.1007/978-3-540-70904-6_6

Coelho, G. L. H. (2018). Which values are similar? Introducing new methodologies to map the structure of human values and value-expressive behaviours, $\mathrm{PhD}$ thesis. Cardiff University. https://orca.cardiff.ac.uk/ 117637/

Coelho, G. L. de H., Hanel, P. H. P., Johansen, M. K., \& Maio, G. R. (2019). Mapping the structure of human values through conceptual representations. European Journal of Personality, 33(1), 34-51. https://doi.org/10. $1002 /$ per. 2170

Eiser, J. R. (1987). The expression of attitude. Springer.

Fischer, R. (2017). From values to behavior and from behavior to values. In S. Roccas \& L. Sagiv, Values and behavior: Taking a cross cultural perspective (pp. 219-235). Springer.

Fiske, S. T., Cuddy, A. J. C., Glick, P., \& Xu, J. (2002). A model of (often mixed) stereotype content: Competence and warmth respectively follow from perceived status and competition. Journal of Personality and Social Psychology, 82(6), 878-902.

Hahn, U., \& Chater, N. (1997). Concepts and similarity. In K. Lamberts \& D. R. Shanks (Eds.), Studies in cognition. Knowledge, concepts and categories (pp. 43-92). The MIT Press.

Haidt, J. (2001). The emotional dog and its rational tail: A social intuitionist approach to moral judgment. Psychological Review, 108(4), 814-834.

Hanel, P. H. P. (2016). Human values and value instantiations: Similarities and differences between countries and their implications [PhD thesis]. Cardiff University. http:// orca.cf.ac.uk/96711/

Hanel, P. H. P., Maio, G. R., \& Manstead, A. S. R. (2019). A new way to look at the data: Similarities between groups of people are large and important. Journal of Personality and Social Psychology, 116(4), 541-562. http://dx.doi.org/10.1037/pspi0000154

Hanel, P. H. P., Maio, G. R., Soares, A. K. S., Vione, K. C., Coelho, G. L. de H., Gouveia, V. V., Patil, A. C., Kamble, S. V., \& Manstead, A. S. R. (2018). Cross-cultural differences and similarities in human value instantiation. Frontiers in Psychology, 9, 849. https://doi.org/10.3389/fpsyg.2018.00849

Hanel, P. H. P., Vione, K. C., Hahn, U., \& Maio, G. R. (2017). Value instantiations: The missing link between values and behavior? In Sonia Roccas \& S. Lilach (Eds.), Values and behaviour: Taking a cross-cultural perspective (pp. 175-190). Springer. https://www. researchgate.net/publication/311651023_Value_instantia tions_The_missing_link_between_values_and_behavior

Hanel, P. H. P., Zacharopoulos, G., Mégardon, G., \& Maio, G. R. (2017). Detecting sinusoidal patterns from circumplex models of psychological constructs. Preprint. https://psyarxiv.com/wh92k/
Hout, M. C., Papesh, M. H., \& Goldinger, S. D. (2013). Multidimensional scaling. Wiley Interdisciplinary Reviews. Cognitive Science, 4(1), 93-103. https://doi. org/10.1002/wcs. 1203

Hubbard, T. L. (2007). What is mental representation? And how does it relate to consciousness? In The concepts of consciousness: Integrating an emerging science (pp. 3761). Imprint Academic.

Jaworska, N., \& Chupetlovska-Anastasova, A. (2009). A review of multidimensional scaling (MDS) and its utility in various psychological domains. Tutorials in Quantitative Methods for Psychology, 5(1), 1-10.

Koch, A., Imhoff, R., Dotsch, R., Unkelbach, C., \& Alves, H. (2016). The ABC of stereotypes about groups: Agency/socioeconomic success, conservativeprogressive beliefs, and communion. Journal of Personality and Social Psychology, 110(5), 675-709. https://doi.org/10.1037/pspa0000046

Kristiansen, C. M., \& Hotte, A. M. (1996). Morality and the self: Implications for when and how of value-attitudebehavior relations. In C. Seligman, J. M. Olson, \& M. P. Zanna, The Ontario Symposium: Vol 8. The psychology of values (pp. 77-106). Lawrence Erlbaum.

Kruglanski, A. W., Köpetz, C., Bélanger, J. J., Chun, W. Y., Orehek, E., \& Fishbach, A. (2013). Features of multifinality. Personality and Social Psychology Review, 17(1), 22-39. https://doi.org/10.1177/1088868312453087

Lee, J. A., Bardi, A., Gerrans, P., Sneddon, J. N., Herk, H. V., Evers, U., \& Schwartz, S. H. (2021). Are valuebehavior relations stronger than previously thought? It depends on value importance. European Journal of Personality. https://doi.org/10.1177/08902070211002965

Maio, G. R. (2010). Mental representations of social values. In M. P. Zanna (Ed.), Advances in experimental social psychology (Vol. 42, pp. 1-43). Academic Press.

Maio, G. R. (2016). The psychology of human values. Psychology Press.

Maio, G. R., Hahn, U., Frost, J.-M., \& Cheung, W.-Y. (2009). Applying the value of equality unequally: Effects of value instantiations that vary in typicality. Journal of Personality and Social Psychology, 97(4), 598-614. https://doi.org/10.1037/a0016683

Maio, G., Haddock, G., \& Verplanken, B. (2018). The psychology of attitudes and attitude change (3rd ed.). SAGE Publications. https://us.sagepub.com/en-us/nam/thepsychology-of-attitudes-and-attitude-change/ book 240842

Maio, G. R., \& Olson, J. M. (1998). Values as truisms: Evidence and implications. Journal of Personality and Social Psychology, 74(2), 294-311. https://doi.org/ 10.1037/0022-3514.74.2.294

Murphy, G. (2004). The big book of concepts. MIT Press.

Oden, G. C. (1987). Concept, knowledge, and thought. Annual Review of Psychology, 38(1), 203-227.

Oksanen, J. (2015). Multivariate analysis of ecological communities in $R$ : Vegan tutorial. http://cc.oulu.fi/ jarioksa/ opetus/metodi/vegantutor.pdf

Oppenheimer, D. M., Meyvis, T., \& Davidenko, N. (2009). Instructional manipulation checks: Detecting satisficing to increase statistical power. Journal of Experimental Social Psychology, 45(4), 867-872. https://doi.org/10. 1016/j.jesp.2009.03.009

Pakizeh, A., Gebauer, J. E., \& Maio, G. R. (2007). Basic human values: Inter-value structure in memory. Journal 
of Experimental Social Psychology, 43(3), 458-465. https://doi.org/10.1016/j.jesp.2006.04.007

Peres-Neto, P. R., \& Jackson, D. A. (2001). How well do multivariate data sets match? The advantages of a Procrustean superimposition approach over the Mantel test. Oecologia, 129(2), 169-178. https://doi.org/10.1007/ s004420100720

R Development Core Team. (2015). R: A language and environment for statistical computing. https://cran.r-project. org/doc/manuals/fullrefman.pdf

Riedl, M., \& Harrison, B. (2016). Using stories to teach human values to artificial agents. AAAI Workshop on AI, Ethics, and Society.

Rokeach, M. (1973). The nature of human values. Free Press.

Schwartz, S. H. (1992). Universals in the content and structure of values: Theoretical advances and empirical tests in 20 countries. Advances in Experimental Social Psychology, 25, 1-65.

Schwartz, S. H. (2007). Value orientations: Measurement, antecedents and consequences across nations. In R. Jowell, C. Roberts, R. Fitzgerald, \& G. Eva (Eds.), Measuring attitudes cross-nationally (pp. 169-203). SAGE Publications, Ltd. http://methods.sagepub.com/ book/measuring-attitudes-cross-nationally/n9.xml

Schwartz, S. H. (2012). An overview of the Schwartz theory of basic values. Online Readings in Psychology and Culture, 2(1). https://doi.org/10.9707/2307-0919.1116

Schwartz, S. H. (2013). Values priorities and behavior: Applying a theory of integrated values systems. In
J. M. Seligman, J. M. Olson, \& M. P. Zanna (Eds.), The psychology of values: The Ontario symposium (pp. 119-144). Psychology Press.

Schwartz, S. H., \& Bardi, A. (2001). Value hierarchies across cultures taking a similarities perspective. Journal of Cross-Cultural Psychology, 32(3), 268-290. https:// doi.org/10.1177/0022022101032003002

Schwartz, S. H., \& Butenko, T. (2014). Values and behavior: Validating the refined value theory in Russia. European Journal of Social Psychology, 44(7), 799-813. https://doi.org/10.1002/ejsp.2053

Schwartz, S. H., \& Sagiv, L. (1995). Identifying culturespecifics in the content and structure of values. Journal of Cross-Cultural Psychology, 26(1), 92-116. https://doi. org/10.1177/0022022195261007

Skimina, E., Cieciuch, J., Schwartz, S. H., Davidov, E., \& Algesheimer, R. (2019). Behavioral signatures of values in everyday behavior in retrospective and real-time selfreports. Frontiers in Psychology, 10, 281. https://doi.org/ 10.3389/fpsyg.2019.00281

Sturrock, K., \& Rocha, J. (2000). A multidimensional scaling stress evaluation table. Field Methods, 12(1), 49-60. https://doi.org/10.1177/1525822X0001200104

Vallacher, R. R., \& Wegner, D. M. (1987). What do people think they're doing? Action identification and human behavior. Psychological Review, 94(1), 3-15. https://doi. org/10.1037/0033-295X.94.1.3

Vallacher, R. R., \& Wegner, D. M. (2014). A theory of action identification. Psychology Press. 